\title{
Regulation of the nitrate reductase operon narKGHJI by the cAMP-dependent regulator GIxR in Corynebacterium glutamicum
}

\begin{abstract}
Correspondence
Hideaki Yukawa

mmg-lab@rite.or.jp
\end{abstract}

Received 12 August 2010

Revised 15 September 2010

Accepted 16 September 2010

\author{
Taku Nishimura, Haruhiko Teramoto, Koichi Toyoda, Masayuki Inui \\ and Hideaki Yukawa
}

\author{
Research Institute of Innovative Technology for the Earth (RITE), 9-2 Kizugawadai, Kizugawa, \\ Kyoto, 619-0292, Japan
}

\section{INTRODUCTION}

Corynebacterium glutamicum, a non-pathogenic high-GC Gram-positive bacterium, has been widely used for industrial production of various amino acids and nucleic acids (Kinoshita, 1985; Terasawa \& Yukawa, 1993). In addition, this species is of increasing interest as a suitable model organism for Corynebacterineae, a suborder of the Actinomycetes, which includes the medically important pathogenic species Corynebacterium diphtheriae and Mycobacterium tuberculosis (Brune et al., 2005; Mishra et al., 2007). C. glutamicum has long been regarded as an aerobic bacterium because there have been few reports of its growth by anaerobic respiration or fermentation, despite the fact that most other coryneform bacteria are facultative anaerobes (Liebl, 2001). Recently, anaerobic growth of $C$. glutamicum by nitrate respiration was reported (Nishimura et al., 2007; Takeno et al., 2007). This property is attributed to the presence of a narKGHJI operon. The narK and narGHJI genes encode a putative nitrate/nitrite transporter and a membrane-bound nitrate reductase complex, respectively. These components of nitrate respiration are conserved in a broad range of bacteria (Moreno-Vivián et al., 1999; Zumft, 1997). Nitrite is excreted from the cell as an end product of anaerobic nitrate reduction in C. glutamicum, and neither nitrate nor nitrite is utilized as the sole nitrogen source for its

Abbreviations: CRP, cAMP receptor protein; EMSA, electrophoretic mobility shift assay. anaerobic or aerobic growth, suggesting that this microorganism lacks an efficient enzymic apparatus to reduce nitrite further (Nishimura et al., 2007; Takeno et al., 2007). This property is distinct from that of well-studied nitratereducing bacteria such as Escherichia coli and Bacillus subtilis, which are able to metabolize nitrate as a nitrogen source to nitrite and further to ammonia (Cole, 1996; Nakano \& Zuber, 1998), and of denitrifiers such as Pseudomonas aeruginosa and Paracoccus denitrificans (Zumft, 1997). Mycobacterium bovis BCG and M. tuberculosis, which are closely related to C. glutamicum, also possess anaerobic nitrate reductase encoded by a narGHJI gene cluster (Sohaskey \& Wayne, 2003; Weber et al., 2000), although these bacteria are unable to grow in the absence of oxygen. The nitrate reductase activity of $M$. tuberculosis cells is strongly induced during chronic infection of mice and under in vitro conditions of gradual oxygen depletion, which are the conditions established to provide a model of the dormant state with latent tuberculosis (Shi et al., 2005; Sohaskey \& Wayne, 2003). It has been suggested that nitrate respiration plays a role in the survival of the pathogen during the inhibition of aerobic respiration (Sohaskey, 2008). Notably, a narG mutant of M. bovis BCG showed reduced virulence and lung damage in both immunocompetent and immunodeficient SCID mice (Fritz et al., 2002; Weber et al., 2000).

Transcription of C. glutamicum narKGHJI is considerably induced by the transition from aerobic to anaerobic nitrate respiratory conditions, as seen in other nitrate-reducing 
bacteria (Nishimura et al., 2007). Our previous study demonstrated that a novel transcriptional regulator, ArnR, directly represses expression of the narKGHJI operon under aerobic conditions (Nishimura et al., 2008). The repression is relieved by a shift to anaerobic nitrate respiratory conditions, resulting in elevated expression of nar genes. In addition to the narKGHJI operon, the hmp gene encoding flavohaemoglobin was identified as an additional target of ArnR (Nishimura et al., 2008). C. glutamicum flavohaemoglobin may be involved in detoxifying nitric oxide during anaerobic nitrate respiration, as has been reported for E. coli (Moreno-Vivián et al., 1999; Poole \& Hughes, 2000; Poole et al., 1996). ArnR's function as an aerobic repressor of the narKGHJI and hmp contrasts with that of the oxygen-sensing global regulator FNR, which activates the expression of many of the genes involved in anaerobic metabolism, including nitrate respiration in E. coli (Bonnefoy \& Demoss, 1994; Gunsalus \& Park, 1994). E. coli FNR constitutes the paradigm of oxygensensing regulators, which have been found in a variety of Gram-negative and a few Gram-positive bacteria (Körner et al., 2003).

GlxR, a member of the CRP/FNR family, was first identified in C. glutamicum as a cAMP-dependent regulator which represses transcription of the glyoxylate shunt genes aceA and aceB (Kim et al., 2004). The GlxR regulon has been predicted to comprise more than 400 genes covering diverse cellular functions (Kohl \& Tauch, 2009; Kohl et al., 2008). Deletion of $g l x R$ indeed results in severe growth defects (Toyoda et al., 2009), implying pleiotropic negative effects. This makes it difficult to examine involvement of GlxR in the transcriptional regulation of individual target genes. Signal transduction and the physiological role of the GlxR-cAMP-mediated regulation are still largely unknown. It was recently reported that GlxR binds to the promoter region of the narKGHJI operon in the presence of cAMP (Kohl et al., 2008). However, in vivo regulation of narKGHJI expression by GlxR remains to be elucidated. How GlxR is involved in anaerobic metabolism of C. glutamicum is of particular interest.

In this study, we investigated the involvement of GlxR and cAMP in the regulation of narKGHJI expression in C. glutamicum. We present in vivo experimental evidence indicating that the positive regulation of narKGHJI expression by GlxR depends on cAMP.

\section{METHODS}

Bacterial strains, media and culture conditions. Strains used in this study are listed in Table 1. C. glutamicum R (FERM P-18976) (Yukawa et al., 2007) and its derivative strains were pre-cultured at $33{ }^{\circ} \mathrm{C}$ overnight in nutrient-rich medium (A medium; Inui et al., $2004 \mathrm{~b})$ with $1 \%(\mathrm{w} / \mathrm{v})$ glucose. The cells were harvested by centrifugation and were subsequently washed once with mineral salts medium (BT medium; Inui et al., 2004b). For aerobic cultivation, the washed cells were grown at $33{ }^{\circ} \mathrm{C}$ in $100 \mathrm{ml}$ BT medium containing
$1 \%(\mathrm{w} / \mathrm{v})$ glucose in a $500 \mathrm{ml}$ flask with vigorous shaking (200 r.p.m.). For anaerobic cultivation, anaerobic BT medium was prepared in advance by bubbling with argon for $5 \mathrm{~min}$. Cells from the same batch as those used for aerobic cultivation were subsequently transferred to the anaerobic BT medium in an anaerobic chamber (Coy Laboratory Products) in which the gas composition was kept constant at $95 \%$ nitrogen and $5 \%$ hydrogen. The cells were grown at $33{ }^{\circ} \mathrm{C}$ in $50 \mathrm{ml} \mathrm{BT}$ medium containing $0.5 \%$ (w/v) glucose in a $50 \mathrm{ml}$ culture bottle with gentle stirring. Potassium nitrate was added to a concentration of $30 \mathrm{mM}$ when necessary. Kanamycin and chloramphenicol were used for cultivation of C. glutamicum strains carrying various plasmids (Table 1), at 50 and $5 \mu \mathrm{g} \mathrm{ml}^{-1}$, respectively.

E. coli JM109 (Takara) was used as a host for all genetic manipulations. E. coli BL21(DE3) (Novagen) was used for protein expression. E. coli strains were cultivated at $37^{\circ} \mathrm{C}$ in $\mathrm{LB}$ medium, supplemented with ampicillin, kanamycin or chloramphenicol (all at $50 \mu \mathrm{g} \mathrm{ml}^{-1}$ ) when necessary.

Construction of arnR and cyaB deletion mutants. Plasmids and primers used in this study are listed in Table 1 and Table 2, respectively.

DNA fragments containing the $a r n R$ and $c y a B$ genes were amplified using primer pairs arnR FWSph/arnR RVXba and cyaB FWXba/cyaB RVSph, respectively. The PCR products were cloned into pCRA725 (Inui et al., 2004a), a suicide vector with the $s a c B$ gene for markerless gene disruption, yielding pCRB622 and pCRC619, respectively. An internal $269 \mathrm{bp}$ fragment of the $a r n R$ gene was removed by inverse PCR using primer pair arnRinv FWBglII/arnRinv RVBglII, with plasmid pCRB622 as a template. Likewise, an internal $1425 \mathrm{bp}$ deletion of the $c y a B$ gene was done using primer pair cyaBinv FWSal/ cyaBinv RVSal, with pCRC619 as a template. The PCR products were digested with BglII and SalI, and self-ligated, yielding pCRB623 and pCRC620, respectively. C. glutamicum $\mathrm{R}$ was transformed by electroporation with pCRB623 and pCRC620, and screening for the deletion mutants was performed as described previously (Inui et al., 2004a). Deletion of the $a r n R$ and $c y a B$ genes was checked by PCR. The mutant strains obtained were designated TN01 $(\triangle a r n R)$ and TN06 $(\Delta c y a B)$.

Construction of narK promoter-lacZ fusions and a plasmid for GIxR overexpression. The DNA fragment containing the narK promoter region was generated by PCR using primer pair pnarK FSmaI/pnarK RSmaI, and cloned upstream of the lac $Z$ gene in pCRA741 (Inui et al., 2007), yielding pCRB626. The direction of the inserts was confirmed by DNA sequencing. Plasmid pCRB626 was isolated as non-methylated DNA from E. coli JM110 for efficient gene introduction into C. glutamicum R (Vertès et al., 1993), and was subsequently integrated into the chromosome of C. glutamicum R, TN01 and TN06 by markerless gene insertion methods, as described previously (Inui et al., 2007), yielding strains TN02, TN04 and TN07, respectively. Mutation in the GlxR binding site was generated by inverse PCR using primer pair glxRmut FW/glxRmut RV, with plasmid pCRB626 as a template. The resulting PCR product was phosphorylated with T4 polynucleotide kinase (Takara Bio) and selfligated to generate pCRB627. Plasmid pCRB627 was integrated into the chromosome of C. glutamicum R and TN01, yielding strains TN03 and TN05, respectively.

Plasmid pCRB621 for GlxR overexpression in the TN02 strain was constructed as described below. The DNA fragment covering the $g l x R$ promoter and coding region was amplified from chromosomal DNA of C. glutamicum R by PCR with primer pair glxR FWEco/glxR RVKpn. The PCR product was cloned into the vector pCRB1, yielding pCRB621. Plasmids pCRB1 and pCRB621 were introduced into the TN02 strain. 
Table 1. Bacterial strains and plasmids used in this study

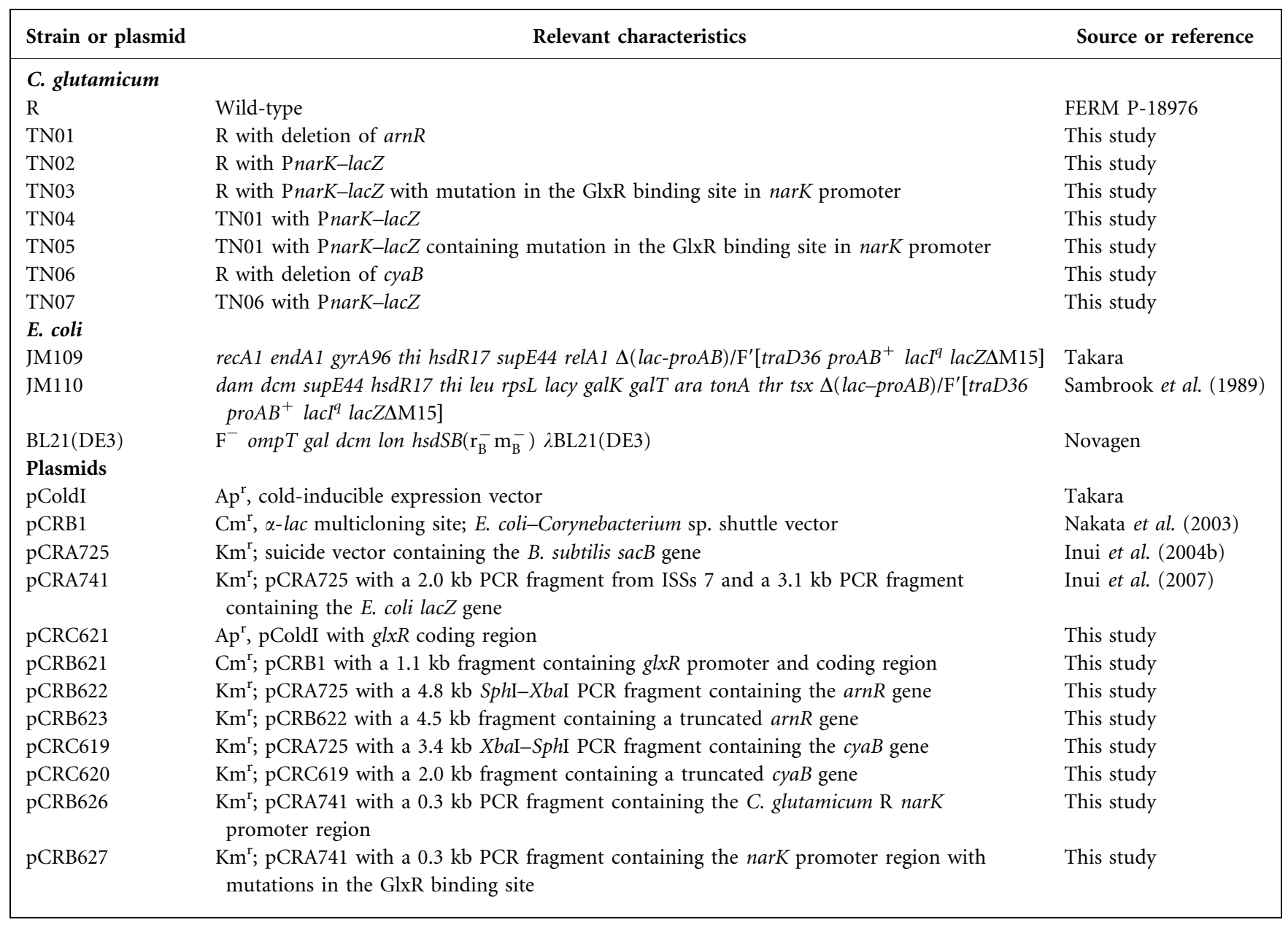

Overproduction and purification of the hexahistidyl-tagged

GIxR protein. The $g l x R$ gene was amplified from chromosomal DNA of C. glutamicum R by PCR with primer pair glxR His FWNde/glxR
His RVSal. The PCR product was cloned into the expression vector pColdI (Takara), yielding pCRC621. E. coli BL21(DE3) transformed with pCRC621 was grown at $37{ }^{\circ} \mathrm{C}$ in $\mathrm{LB}$ medium to an $\mathrm{OD}_{610}$ of 0.5 .

Table 2. Oligonucleotide primers used in this study

\begin{tabular}{|ll|}
\hline Name & \multicolumn{1}{c|}{ Sequence $\mathbf{( 5}^{\prime} \mathbf{3}^{\prime} \mathbf{)}$} \\
\hline $\begin{array}{l}\text { glxR His FWNde } \\
\text { glxR His RVSal } \\
\text { pnarK FWSmaI } \\
\text { pnarK RVSmaI }\end{array}$ & GAGAAAACATATGGAAGGTGTACAGGAGATCC \\
glxRmut FW & GCGCTTGTCGACTTATCGAGCGCGACGTGCCAAATG \\
glxRmut RV & TCCCCCGGGCATCAATTTCTCCTCAAGTGG \\
glxR FWEco & TGCATAAACCCCATTACGACACAA \\
glxR RVKpn & GGTAAACGAATTTGTGCTTATATC \\
arnR FWSph & GGAATTCGCTGCCTAAATAAGGGTTATG \\
arnR RVXba & GGGGTACCGTGGCTATTCGTTACCTGCA \\
arnRinv FWBglII & ACATGCATGCGGATGCGCACCCATACTGG \\
arnRinv RVBglII & GCTCTAGAGTCTATAGTGGCTAGGTACC \\
cyaB FWXba & GAAGATCTGACGATGACGCAATGCACAA \\
cyaB RVSph & GAAGATCTTGGGCTGAGTTTGTAGGACT \\
cyaBinv FWSal & CGATCTAGATTCTTACGGCTTCGAGTTCGGCTTC \\
cyaBinv RVSal & GAGCGGCATGCCAAGGAAGCAGCAGATGGCGGAG \\
\hline
\end{tabular}


The culture was incubated for $30 \mathrm{~min}$ at $15{ }^{\circ} \mathrm{C}$, then expression of the His-tagged GlxR was induced by addition of $0.5 \mathrm{mM}$ IPTG. The culture was shaken overnight at $15{ }^{\circ} \mathrm{C}$. The cells were harvested by centrifugation and frozen at $-80{ }^{\circ} \mathrm{C}$ until ready for use. The Histagged protein was purified by affinity chromatography on Ni-NTA agarose (Qiagen) according to the instruction manual. For desalting, the eluted protein was loaded onto a PD-10 column (GE Healthcare Bioscience) and eluted with buffer A $(50 \mathrm{mM}$ Tris/ $\mathrm{HCl}, \mathrm{pH} 7.5$, $10 \mathrm{mM} \mathrm{MgCl} 2,1 \mathrm{mM}$ EDTA and $1 \mathrm{mM}$ DTT). The concentration of the purified protein was determined by a Bio-Rad protein assay using BSA as a standard.

Electrophoretic mobility shift assay (EMSA). The wild-type DNA probe containing the narK promoter region was amplified by PCR using primer pair pnarK FSmaI/pnarK RSmaI and C. glutamicum $\mathrm{R}$ genomic DNA as a template. The DNA probe with mutation in the GlxR binding site was amplified by PCR using plasmid pCRB627 as a template.

Binding reactions were performed using a solution containing $10 \mathrm{mM}$ Tris/HCl (pH 7.4), $50 \mathrm{mM} \mathrm{KCl}$ and $0.1 \mathrm{mM}$ EDTA. A total of $10 \mathrm{nM}$ specific DNA fragment and 0-800 $\mathrm{nM}$ (0-80 molar excess) purified GlxR-His ${ }_{6}$ protein were added to a final volume of $10 \mu \mathrm{l}$ and incubated at room temperature for $15 \mathrm{~min}$. The samples were immediately loaded onto a $5 \%$ non-denaturing polyacrylamide gel and electrophoresed at $150 \mathrm{~V}$ in $0.5 \times$ TBE buffer $(44.5 \mathrm{mM}$ Tris base, $44.5 \mathrm{mM}$ boric acid, $1 \mathrm{mM}$ EDTA). The gel was stained using an EMSA kit (Invitrogen).

$\boldsymbol{\beta}$-Galactosidase assay. Exponentially growing C. glutamicum cells harbouring the narK promoter-lac $Z$ fusion were harvested, and washed with Z-buffer (Miller, 1972). Toluene treatment and $\beta$ galactosidase assays using $o$-nitrophenyl $\beta$-D-galactopyranoside were performed as described by Miller (1972). The values presented are the means of at least three independent experiments.

Nitrate reductase enzyme assay. To prepare crude cell extracts for nitrate reductase enzyme assay, exponentially growing $C$. glutamicum cells were harvested, and washed with phosphate buffer ( $\mathrm{pH}$ 7.2). The pellet was resuspended in the same buffer. A $1 \mathrm{ml}$ volume of the cell suspension was mixed with $0.5 \mathrm{~g} 0.1 \mathrm{~mm}$ zirconia/silica beads. The cells were mechanically disrupted with a Multi-Beads Shocker (Yasui Kikai) at 2500 r.p.m. for 10 periods of $30 \mathrm{~s}$ at $4{ }^{\circ} \mathrm{C}$. The resultant lysate was centrifuged for $10 \mathrm{~min}$ at $2000 \mathrm{~g}$ and $4{ }^{\circ} \mathrm{C}$ and the supernatant was used for nitrate reductase enzyme assay. Nitrate reductase activity was measured as described previously (MacGregor \& Schnaitman, 1974). Nitrite concentration was determined by using an $\mathrm{NO}_{2} / \mathrm{NO}_{3}$ Assay kit-C II (Colorimetric) - Griess Reagent kit (Dojindo).

\section{RESULTS}

\section{A consensus motif essential for CAMP-dependent specific binding of GIXR to the narK promoter region}

In vitro cAMP-dependent binding of GlxR to the $40 \mathrm{bp}$ upstream region of the narKGHJI operon was shown previously, and a putative $16 \mathrm{bp}$ consensus motif for the GlxR binding was identified in its upstream region (Kohl et al., 2008). To verify specific interaction of GlxR with the putative binding site (AGTGAATTACGACACA) in the narK promoter region, we performed mutational analysis using EMSAs. In the presence of cAMP, purified GlxR bound to the wild-type narK promoter region, which covers the region -199 through +82 bp relative to the transcriptional start site of the narKGHJI operon, while binding was not detected in the absence of cAMP (Fig. 1). The cAMP-dependent interaction was completely inhibited by introduction of a mutation in the putative binding motif (ACCCCATTACGACACA), indicating that the motif is essential for GlxR binding.

\section{GIxR is involved in activation of narKGHJI expression}

It was previously reported that growth of a $g l x R$ deletion mutant is too slow to be analysed (Toyoda et al., 2009). To verify the involvement of GlxR in expression of the narKGHJI operon, the influence on promoter activity of mutation of the GlxR binding motif, as tested in EMSAs, was examined using a lac $Z$ reporter system. The wild-type narK promoter-lacZ fusion (PnarK-lacZ) and the mutant $\mathrm{P}$ narK-lac $Z$ were integrated into the chromosome of the $C$. glutamicum wild-type strain, yielding strains TN02 and

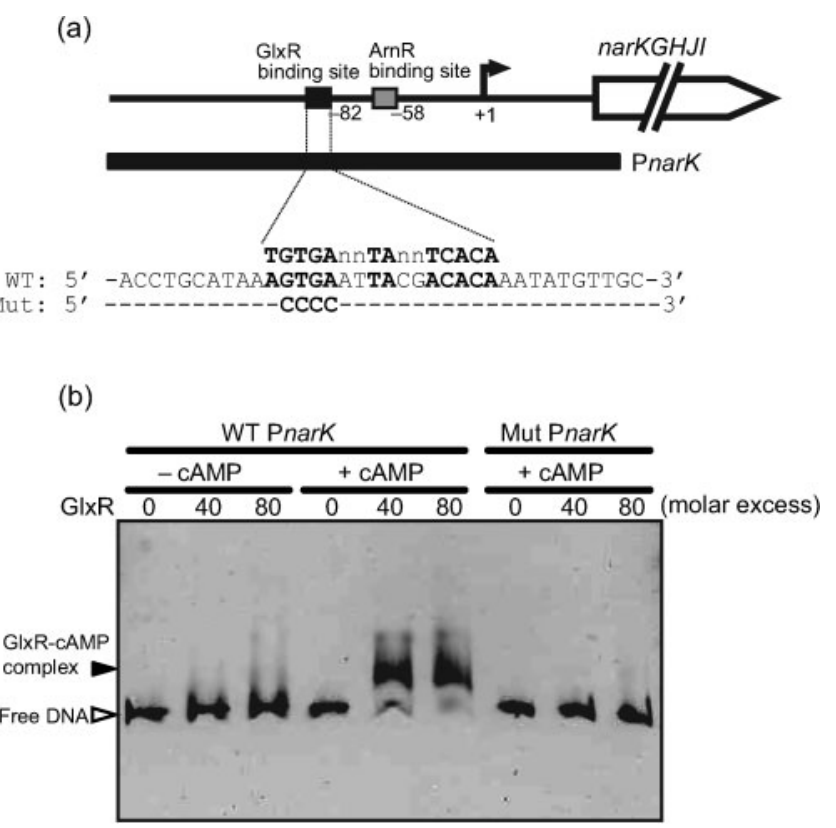

Fig. 1. (a) Genomic locus of the narK promoter region with the GlxR and ArnR binding sites, and the DNA probe covering the narK promoter region for EMSAs (PnarK). Numbers adjacent to the GlxR and ArnR binding sites indicate the first position of the binding site relative to the transcriptional start site $(+1)$. The $\mathrm{G} \mid x \mathrm{R}$ consensus binding site (TGTGAnnTAnnTCACA) and the mutation (Mut) introduced into the DNA probe are shown above and below the wild-type (WT) sequence, respectively. (b) EMSAs were carried out using the wild-type and the mutant DNA probes (WT PnarK and Mut PnarK, respectively) and purified His-tagged GlxR protein with $(+)$ or without $(-) 0.2 \mathrm{mM}$ cAMP. GlxR protein was added at a molar excess of 0,40 or 80 to $10 \mathrm{nM}$ DNA probe. Free DNA and GIxR-cAMP complex are indicated with open and filled arrowheads, respectively. 
Table 3. Involvement of the GIxR binding site and cyaB gene in regulation of narKGHJI expression

The wild-type and its $\operatorname{arnR}$ and $c y a B$ deletion mutants $(\triangle a r n R$ and $\Delta c y a B)$, expressing wild-type or mutant $n a r K$ promoter-lacZ gene fusion (WT PnarK-lacZ or Mut PnarK-lacZ), were grown to exponential phase in BT minimal medium under aerobic or anaerobic nitrate respiratory conditions as described in Methods. They were then subjected to $\beta$-galactosidase assay. The results are reported as means \pm SD of at least three independent experiments.

\begin{tabular}{|c|c|c|c|}
\hline \multirow[t]{2}{*}{ Strain (relevant genotype) } & \multirow[t]{2}{*}{ Reporter gene fusion } & \multicolumn{2}{|c|}{$\beta$-Galactosidase activity (Miller units) } \\
\hline & & Aerobic & $\begin{array}{c}\text { Anaerobic nitrate } \\
\text { respiration }\end{array}$ \\
\hline TN02 (wild-type) & WT PnarK-lacZ & $8.6 \pm 0.6$ & $97.8 \pm 9.2$ \\
\hline TN03 (wild-type) & Mut PnarK-lacZ & $1.5 \pm 0.4$ & $32.8 \pm 2.3$ \\
\hline TN04 $(\Delta a r n R)$ & WT PnarK-lacZ & $54.9 \pm 4.9$ & $147.5 \pm 9.3$ \\
\hline TN05 $(\Delta a r n R)$ & Mut PnarK-lacZ & $23.3 \pm 1.0$ & $68.5 \pm 0.6$ \\
\hline TN07 $(\Delta c y a B)$ & WT PnarK-lacZ & $<0.1$ & $14.3 \pm 3.3$ \\
\hline
\end{tabular}

TN03, respectively. $\beta$-Galactosidase activity of these strains grown under aerobic and anaerobic nitrate respiratory conditions was measured (Table 3). The PnarK-lacZ expression observed in the TN02 strain was markedly higher under anaerobic nitrate respiratory conditions than under aerobic conditions. The induction of narK promoter activity in response to anaerobiosis is consistent with findings of a previous study (Nishimura et al., 2008). Under anaerobic conditions, the mutant PnarK-lacZ expression observed in the TN03 strain was significantly lower than the wild-type PnarK-lacZ expression observed in the TN02 strain. Under aerobic conditions, the narK promoter activity of the TN02 strain was repressed at a low basal level; however, mutation of the GlxR binding site resulted in further decreased activity, as seen in the TN03 strain. Effects of the GlxR binding-site mutation on the narK promoter activity was also investigated in the $\operatorname{arn} R$ deletion mutant strains $(\triangle a r n R)$ (Table 3$)$. The wild-type narK promoter activity was derepressed by $a r n R$ disruption especially under aerobic conditions, as seen in the TN04 strain. This is consistent with our previous study indicating that ArnR functions as an aerobic repressor of the narKGHJI operon (Nishimura et al., 2008). The mutant PnarK-lacZ expression of the $\Delta a r n R$ strain (TN05) was significantly lower than the wild-type promoter-lac $Z$ expression of the $\triangle a r n R$ strain (TN04) under both aerobic and anaerobic nitrate respiratory conditions. These results suggest that binding of GlxR is responsible for upregulation of the narK promoter activity, regardless of ArnR function.

To buttress the role of GlxR as an activator of narKGHJI expression, the influence of GlxR overexpression on narK promoter activity was examined. We introduced a multicopy plasmid carrying the $g l x R$ promoter and coding region (pCRB621) into the wild-type strain expressing PnarK-lacZ (TN02), and measured its $\beta$-galactosidase activity (Table 4). The narK promoter activity of the TN02 strain carrying pCRB621 was approximately 1.4- and 2.0-fold higher than that of a strain carrying empty vector (pCRB1) under aerobic and anaerobic nitrate respiratory conditions, respectively. This result is consistent with that observed with mutational analysis of the GlxR binding site in the narK promoter.

\section{An adenylate cyclase gene, cyaB, is involved in the activation of narKGHJ expression}

EMSAs showed that GlxR is unable to interact with the binding motif in the narK promoter region without cAMP in vitro (Fig. 1). The intracellular cAMP level in $C$. glutamicum has been previously shown to decrease upon deletion of the adenylate cyclase gene cyaB (Cha et al., 2010). Here, we examined the effects of $c y a B$ deletion on narK promoter activity. Wild-type PnarK-lacZ was integrated into the chromosome of a $c y a B$ deletion strain $(\Delta c y a B)$, and its $\beta$-galactosidase activity measured (Table 3 ). The narK promoter activity of the $\Delta c y a B$ strain (TN07) was markedly lower than that of the wild-type strain (TN02) under aerobic and anaerobic nitrate respiratory conditions. The effect of $c y a B$ deletion on narK promoter activity was relatively larger than that of mutation of the GlxR binding site. It should be noted that cyaB deletion

Table 4. Effects of overexpression of the $g / x R$ gene on narK promoter activity

Strain TN02 harbouring control vector pCRB1 or plasmid pCRB621 for GlxR overexpression was grown to exponential phase in BT minimal medium under aerobic or anaerobic nitrate respiratory conditions. $\beta$-Galactosidase activity was then determined. The data are means \pm SD of at least three independent experiments.

\begin{tabular}{|lcc|}
\hline Strain (plasmid) & $\boldsymbol{\beta}$-Galactosidase activity (Miller units) \\
\cline { 2 - 3 } & Aerobic & $\begin{array}{c}\text { Anaerobic nitrate } \\
\text { respiration }\end{array}$ \\
\hline TN02 (pCRB1) & $7.8 \pm 1.8$ & $58.7 \pm 11.2$ \\
TN02 (pCRB621) & $11.0 \pm 0.6$ & $117.9 \pm 8.8$ \\
\hline
\end{tabular}


Table 5. Influence of $c y a B$ deletion on nitrate reductase activity

The wild-type $\mathrm{R}$ strain and its $c y a B$ deletion mutant $(\Delta c y a B$, strain TN06) were grown to the exponential phase in BT minimal medium under aerobic and anaerobic nitrate respiratory conditions, and their nitrate reductase activity was determined as described in Methods. The data are the means $\pm S D$ of at least three independent experiments.

\begin{tabular}{|c|c|c|}
\hline \multirow[t]{2}{*}{$\begin{array}{l}\text { Strain (relevant } \\
\text { genotype) }\end{array}$} & \multicolumn{2}{|c|}{$\begin{array}{l}\text { Nitrate reductase activity [nmol } \mathrm{min}^{-1} \\
\left.\qquad(\mathrm{mg} \text { protein })^{-1}\right]\end{array}$} \\
\hline & Aerobic & $\begin{array}{l}\text { Anaerobic nitrate } \\
\text { respiration }\end{array}$ \\
\hline R (wild-type) & $4.8 \pm 1.2$ & $17.1 \pm 0.9$ \\
\hline TN06 $(\Delta c y a B)$ & $3.0 \pm 0.6$ & $9.1 \pm 0.4$ \\
\hline
\end{tabular}

may affect a variety of cAMP-dependent cellular functions, including the wide physiological role of GlxR.

To confirm the positive effect of cAMP on the narK promoter activity, nitrate reductase enzyme (NAR) activity of the $\triangle c y a B$ strain was evaluated (Table 5). The NAR activity of the $\triangle c y a B$ strain (TN06) was $40-50 \%$ lower than that of the wild-type strain under aerobic and anaerobic nitrate respiratory conditions, although the influence of $c y a B$ disruption was relatively weak when compared with that on the narK promoter activity (Table 3 ). The positive regulation of the promoter activity by GlxR and cAMP, in conjunction with the in vitro GlxR binding to the narK promoter region only in the presence of cAMP, indicates that in vivo activation of the narKGHJI expression by GlxR is cAMP-dependent.

\section{DISCUSSION}

This study showed that mutation of a putative GlxR binding motif located in the narK promoter region of $C$. glutamicum resulted in inhibition of the in vitro binding of GlxR and a significant decrease in the narK promoter activity in vivo. Furthermore, deletion of the cyaB gene caused considerable reduction of the narK promoter activity. These results indicate that the CRP-type regulator GlxR positively regulates narKGHJI expression in a cAMPdependent manner in C. glutamicum. This is supported by the finding that GlxR overexpression using a multi-copy plasmid enhanced the narK promoter activity in trans. The highly induced narK promoter activity under anaerobic conditions was shown to be repressed several-fold by GlxR binding site mutation and $c y a B$ disruption, suggesting that GlxR contributes to the maintenance of high-level narKGHJI expression. However, narKGHJI expression repressed at a low basal level under aerobic conditions was further decreased by those genetic modifications. The transcriptional regulator ArnR strongly represses narKGHJI expression under aerobic conditions (Nishimura et al.,
2008). It is noted that the narK promoter activity was clearly repressed by the GlxR binding motif mutation even under conditions where the repression is released by deletion of the $\operatorname{arnR}$ gene. Therefore, ArnR plays a key regulatory role in response to anaerobiosis, and GlxR probably acts as an activator of the narKGHJI operon irrespective of the ArnR function. Concerning residual induction of narKGHJI expression in the $\operatorname{arnR}$ deletion mutant (Table 3), it is possible that additional regulators are involved in anaerobic induction of expression. Consequently, it is suggested that GlxR is not directly associated with the sensing mechanism for the anaerobiosis-responsive induction of narKGHJI expression. The regulation of narKGHJI expression by ArnR and CRP-type GlxR regulators in C. glutamicum is quite different from the well-known FNR-dependent anaerobic induction of the nitrate reductase gene expression in E. coli and B. subtilis (Bonnefoy \& Demoss, 1994; Reents et al., 2006). In Shewanella oneidensis MR-1, a metal reducer that uses more than 14 terminal electron acceptors for respiration, instead of EtrA (an FNR homologue), CRP is involved in positive regulation of a variety of anaerobic respiration including nitrate, DMSO, fumarate and $\mathrm{Fe}(\mathrm{III})$ reduction (Saffarini et al., 2003). Expression of the relevant genes has been suggested to be anaerobically induced by CRP in a cAMP-dependent manner (Charania et al., 2009), although control of the intracellular cAMP level in response to anaerobiosis remains unclear.

The degree of anaerobic induction of C. glutamicum nitrate reductase activity was relatively small compared with that of the narKGHJI transcription level. It has been previously reported that nitrate reductase activity was not influenced by the oxygen and nitrate levels, although aerobically growing C. glutamicum cells exhibited no significant nitrite production in the presence of oxygen (Takeno et al., 2007). This may be explained by preferential electron flow to oxygen due to the higher electron-accepting affinity of cytochrome oxidase than nitrate reductase enzymes. The current findings suggest that unknown factors at posttranscriptional and translational levels in addition to the transcriptional level affect nitrate reductase expression in C. glutamicum. It is possible that C. glutamicum nitrate reductase may function to some extent using nitrate as an additional or alternative electron acceptor under low oxygen tension as well as anaerobic conditions. In $M$. tuberculosis, narGHJI expression is constitutive irrespective of the presence of oxygen. Instead, the whole-cell nitrate reductase activity is regulated by control of the transcriptional level of the putative nitrate/nitrite transporter gene narK2 (Sohaskey \& Wayne, 2003). In contrast to $C$. glutamicum, $M$. tuberculosis is able to utilize nitrate aerobically as a nitrogen source (Malm et al., 2009). Further investigation is required to understand the physiological role of nitrate reductase under changing environmental conditions in C. glutamicum.

The intracellular cAMP level of C. glutamicum during aerobic growth is significantly higher in the presence of 
glucose than that in acetate-grown cells (Kim et al., 2004), which is opposite to the situation in E. coli (Crother \& Steitz, 1992). GlxR-cAMP is presumably involved in acetate-responsive transcriptional induction of the glyoxylate bypass genes aceA and aceB, and that of the TCA cycle genes $s d h C A B$ and gltA (Bussmann et al., 2009; Kim et al., 2004; van Ooyen et al., 2010), although the regulatory mechanism of cAMP level is still unclear. It should be noted that GlxR-cAMP probably regulates expression of genes with a variety of cellular functions, including metabolism not only of carbon but also of nitrogen and phosphate, iron-sulfur homeostasis and the stress response (Kohl \& Tauch, 2009; Panhorst et al., 2010). Therefore, it remains unknown what signal GlxR-cAMP responds to and what regulatory role GlxR-cAMP plays in $C$. glutamicum. In this context, it should be noted that the $c y a B$ disruptant still has a certain level of intracellular cAMP, as reported in a recent study (Cha et al., 2010), and that the phenotypes of the $g l x R$ and $c y a B$ deletion mutants are not comparable (Cha et al., 2010; Park et al., 2010). Further studies on the regulatory mechanism of GlxRcAMP may provide insight into new aspects of the role of cAMP in global genetic regulation in bacteria.

\section{ACKNOWLEDGEMENTS}

We thank Crispinus A. Omumasaba (Research Institute of Innovative Technology for the Earth) for critical reading of the manuscript. This study was partially supported by a grant from the New Energy and Industrial Technology Development Organization (NEDO), Japan.

\section{REFERENCES}

Bonnefoy, V. \& Demoss, J. A. (1994). Nitrate reductases in Escherichia coli. Antonie van Leeuwenhoek 66, 47-56.

Brune, I., Brinkrolf, K., Kalinowski, J., Püehler, A. \& Tauch, A. (2005). The individual and common repertoire of DNA-binding transcriptional regulators of Corynebacterium glutamicum, Corynebacterium efficiens, Corynebacterium diphtheriae and Corynebacterium jeikeium deduced from the complete genome sequences. BMC Genomics 6, 86 .

Bussmann, M., Emer, D., Hasenbein, S., Degraf, S., Eikmanns, B. J. \& Bott, M. (2009). Transcriptional control of the succinate dehydrogenase operon $s d h C A B$ of Corynebacterium glutamicum by the cAMP-dependent regulator GlxR and the LuxR-type regulator RamA. J Biotechnol 143, 173-182.

Cha, P. H., Park, S. Y., Moon, M. W., Subhadra, B., Oh, T. K., Kim, E., Kim, J. F. \& Lee, J. K. (2010). Characterization of an adenylate cyclase gene $(c y a B)$ deletion mutant of Corynebacterium glutamicum ATCC 13032. Appl Microbiol Biotechnol 85, 1061-1068.

Charania, M. A., Brockman, K. L., Zhang, Y., Banerjee, A., Pinchuk, G. E., Fredrickson, J. K., Beliaev, A. S. \& Saffarini, D. A. (2009). Involvement of a membrane-bound class III adenylate cyclase in regulation of anaerobic respiration in Shewanella oneidensis MR-1. J Bacteriol 191, 4298-4306.

Cole, J. (1996). Nitrate reduction to ammonia by enteric bacteria: redundancy, or a strategy for survival during oxygen starvation? FEMS Microbiol Lett 136, 1-11.

Crother, D. M. \& Steitz, T. A. (1992). Transcriptional activation by Escherichia coli Crp protein. In Transcriptional Regulation, pp.
501-534. Edited by S. L. Mcknight \& K. R. Yamamoto. Cold Spring Harbor, NY: Cold Spring Harbor Laboratory.

Fritz, C., Maass, S., Kreft, A. \& Bange, F. C. (2002). Dependence of Mycobacterium bovis BCG on anaerobic nitrate reductase for persistence is tissue specific. Infect Immun 70, 286-291.

Gunsalus, R. P. \& Park, S. J. (1994). Aerobic-anaerobic gene regulation in Escherichia coli: control by the ArcAB and Fnr regulons. Res Microbiol 145, 437-450.

Inui, M., Kawaguchi, H., Murakami, S., Vertès, A. A. \& Yukawa, H. (2004a). Metabolic engineering of Corynebacterium glutamicum for fuel ethanol production under oxygen-deprivation conditions. J Mol Microbiol Biotechnol 8, 243-254.

Inui, M., Murakami, S., Okino, S., Kawaguchi, H., Vertès, A. A. \& Yukawa, H. (2004b). Metabolic analysis of Corynebacterium glutamicum during lactate and succinate productions under oxygen deprivation conditions. J Mol Microbiol Biotechnol 7, 182-196.

Inui, M., Suda, M., Okino, S., Nonaka, H., Puskas, L. G., Vertès, A. A. \& Yukawa, H. (2007). Transcriptional profiling of Corynebacterium glutamicum metabolism during organic acid production under oxygen deprivation conditions. Microbiology 153, 2491-2504.

Kim, H. J., Kim, T. H., Kim, Y. \& Lee, H. S. (2004). Identification and characterization of $g l x R$, a gene involved in regulation of glyoxylate bypass in Corynebacterium glutamicum. J Bacteriol 186, 3453-3460.

Kinoshita, S. (1985). Glutamic acid bacteria. In Biology of Industrial Microorganisms, pp. 115-146. Edited by A. L. Demain \& N. A. Solomon. London: Benjamin Cummings.

Kohl, T. A. \& Tauch, A. (2009). The GlxR regulon of the amino acid producer Corynebacterium glutamicum: Detection of the corynebacterial core regulon and integration into the transcriptional regulatory network model. J Biotechnol 143, 239-246.

Kohl, T. A., Baumbach, J., Jungwirth, B., Pühler, A. \& Tauch, A. (2008). The GlxR regulon of the amino acid producer Corynebacterium glutamicum: in silico and in vitro detection of DNA binding sites of a global transcription regulator. J Biotechnol 135, 340-350.

Körner, H., Sofia, H. J. \& Zumft, W. G. (2003). Phylogeny of the bacterial superfamily of Crp-Fnr transcription regulators: exploiting the metabolic spectrum by controlling alternative gene programs. FEMS Microbiol Rev 27, 559-592.

Liebl, W. (2001). Corynebacterium nonmedical. In The Prokaryotes, pp. 796-818. Edited by M. Dworkin, S. Falkow, E. Rosenberg, K.-H. Schleifer \& E. Stackebrandt. New York: Springer.

MacGregor, C. H. \& Schnaitman, C. A. (1974). Nitrate reductase in $E$. coli: properties of the enzyme and in vitro reconstitution from enzyme-deficient mutants. J Supramol Struct 2, 715-727.

Malm, S., Tiffert, Y., Micklinghoff, J., Schultze, S., Joost, I., Weber, I., Horst, S., Ackermann, B., Schmidt, M. \& other authors (2009). The roles of the nitrate reductase NarGHJI, the nitrite reductase NirBD and the response regulator $\mathrm{GlnR}$ in nitrate assimilation of Mycobacterium tuberculosis. Microbiology 155, 1332-1339.

Miller, J. H. (1972). Experiments in Molecular Genetics. Cold Spring Harbor, NY: Cold Spring Harbor Laboratory.

Mishra, A. K., Alderwick, L. J., Rittmann, D., Tatituri, R. V., Nigou, J., Gilleron, M., Eggeling, L. \& Besra, G. S. (2007). Identification of an $\alpha(1 \rightarrow 6)$ mannopyranosyltransferase (MptA), involved in Corynebacterium glutamicum lipomanann biosynthesis, and identification of its orthologue in Mycobacterium tuberculosis. Mol Microbiol 65, 15031517.

Moreno-Vivián, C., Cabello, P., Martínez-Luque, M., Blasco, R. \& Castillo, F. (1999). Prokaryotic nitrate reduction: molecular properties and functional distinction among bacterial nitrate reductases. J Bacteriol 181, 6573-6584. 
Nakano, M. M. \& Zuber, P. (1998). Anaerobic growth of a "strict aerobe" (Bacillus subtilis). Annu Rev Microbiol 52, 165-190.

Nakata, K., Inui, M., Kos, P. B., Vertès, A. A. \& Yukawa, H. (2003). Vectors for the genetics engineering of corynebacteria. In Fermentaion Biotechnology, ACS Symposium Series 862, pp. 175-191. Edited by B. C. Saha. Washington, DC: American Chemical Society.

Nishimura, T., Vertès, A. A., Shinoda, Y., Inui, M. \& Yukawa, H. (2007). Anaerobic growth of Corynebacterium glutamicum using nitrate as a terminal electron acceptor. Appl Microbiol Biotechnol 75, 889-897.

Nishimura, T., Teramoto, H., Vertès, A. A., Inui, M. \& Yukawa, H. (2008). ArnR, a novel transcriptional regulator, represses expression of the narKGHJI operon in Corynebacterium glutamicum. J Bacteriol 190, 3264-3273.

Panhorst, M., Sorger-Herrmann, U. \& Wendisch, V. F. (2010). The pstSCAB operon for phosphate uptake is regulated by the global regulator GlxR in Corynebacterium glutamicum. J Biotechnol July 16 [Epub ahead of print].

Park, S. Y., Moon, M. W., Subhadra, B. \& Lee, J. K. (2010). Functional characterization of the $g l x R$ deletion mutant of Corynebacterium glutamicum ATCC 13032: involvement of GlxR in acetate metabolism and carbon catabolite repression. FEMS Microbiol Lett 304, 107-115.

Poole, R. K. \& Hughes, M. N. (2000). New functions for the ancient globin family: bacterial responses to nitric oxide and nitrosative stress. Mol Microbiol 36, 775-783.

Poole, R. K., Anjum, M. F., Membrillo-Hernández, J., Kim, S. O., Hughes, M. N. \& Stewart, V. (1996). Nitric oxide, nitrite, and Fnr regulation of $h m p$ (flavohemoglobin) gene expression in Escherichia coli K-12. J Bacteriol 178, 5487-5492.

Reents, H., Münch, R., Dammeyer, T., Jahn, D. \& Härtig, E. (2006). The Fnr regulon of Bacillus subtilis. J Bacteriol 188, 1103-1112.

Saffarini, D. A., Schultz, R. \& Beliaev, A. (2003). Involvement of cyclic AMP (cAMP) and cAMP receptor protein in anaerobic respiration of Shewanella oneidensis. J Bacteriol 185, 3668-3671.

Sambrook, J., Fritsch, E. F. \& Maniatis, T. (1989). Molecular Cloning: a Laboratory Manual, 2nd edn. Cold Spring Harbor, NY: Cold Spring Harbor Laboratory.

Shi, L., Sohaskey, C. D., Kana, B. D., Dawes, S., North, R. J., Mizrahi, V. \& Gennaro, M. L. (2005). Changes in energy metabolism of Mycobacterium tuberculosis in mouse lung and under in vitro conditions affecting aerobic respiration. Proc Natl Acad Sci U S A 102, 15629-15634.

Sohaskey, C. D. (2008). Nitrate enhances the survival of Mycobacterium tuberculosis during inhibition of respiration. J Bacteriol 190, 2981-2986.

Sohaskey, C. D. \& Wayne, L. G. (2003). Role of narK2X and narGHJI in hypoxic upregulation of nitrate reduction by Mycobacterium tuberculosis. J Bacteriol 185, 7247-7256.

Takeno, S., Ohnishi, J., Komatsu, T., Masaki, T., Sen, K. \& Ikeda, M. (2007). Anaerobic growth and potential for amino acid production by nitrate respiration in Corynebacterium glutamicum. Appl Microbiol Biotechnol 75, 1173-1182.

Terasawa, M. \& Yukawa, H. (1993). Industrial production of biochemicals by native immobilization. In Industrial Application of Immobilized Biocatalists, pp. 37-52. Edited by T. Kobayashi. New York: Dekker.

Toyoda, K., Teramoto, H., Inui, M. \& Yukawa, H. (2009). Involvement of the LuxR-type transcriptional regulator RamA in regulation of expression of the gapA gene, encoding glyceraldehyde-3-phosphate dehydrogenase of Corynebacterium glutamicum. J Bacteriol 191, 968977.

van Ooyen, J., Emer, D., Bussmann, M., Bott, M., Eikmanns, B. J. \& Eggeling, L. (2010). Citrate synthase in Corynebacterium glutamicum is encoded by two gltA transcripts which are controlled by RamA, RamB, and GlxR. J Biotechnol July 12 [Epub ahead of print].

Vertès, A. A., Inui, M., Kobayashi, M., Kurusu, Y. \& Yukawa, H. (1993). Presence of $\mathrm{mrr}$ - and $\mathrm{mcr}$-like restriction systems in coryneform bacteria. Res Microbiol 144, 181-185.

Weber, I., Fritz, C., Ruttkowski, S., Kreft, A. \& Bange, F. C. (2000). Anaerobic nitrate reductase (narGHJI) activity of Mycobacterium bovis BCG in vitro and its contribution to virulence in immunodeficient mice. Mol Microbiol 35, 1017-1025.

Yukawa, H., Omumasaba, C. A., Nonaka, H., Kos, P., Okai, N., Suzuki, N., Suda, M., Tsuge, Y., Watanabe, J. \& other authors (2007). Comparative analysis of the Corynebacterium glutamicum group and complete genome sequence of strain R. Microbiology 153, 1042-1058.

Zumft, W. G. (1997). Cell biology and molecular basis of denitrification. Microbiol Mol Biol Rev 61, 533-616.

Edited by: J. Moir 